\title{
Rectificador monofásico para UPS con reducción de contenido armónico en la corriente de entrada
}

\author{
UPS single-phase rectifier with reduction of current \\ harmonic content
}

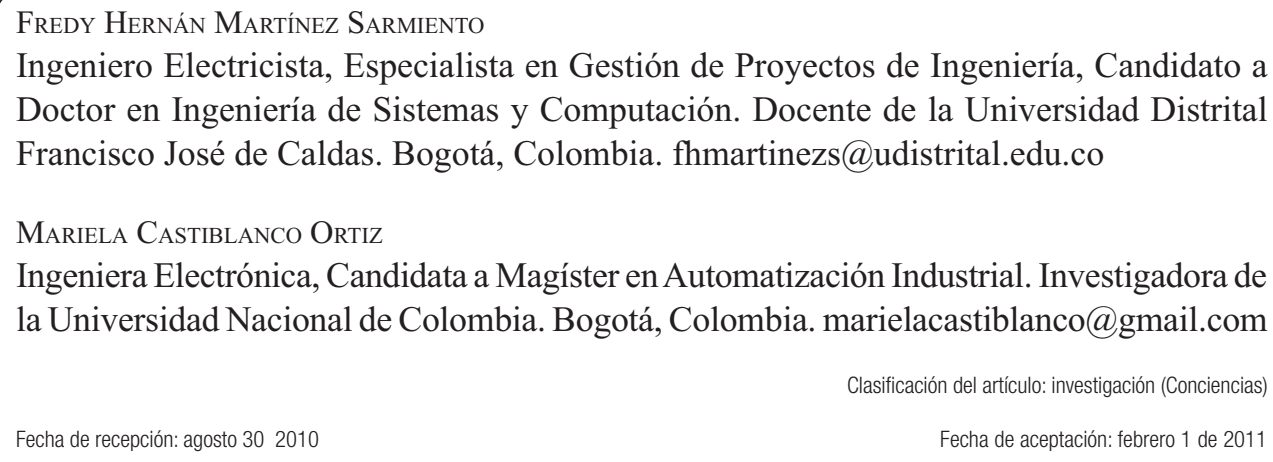

Palabras clave: Control por histéresis, Distorsión de corriente, Rectificador activo.

Key words: Hysteresis control, Current distortion, Active rectifier.

\section{RESUMEN}

Este artículo presenta el diseño, desarrollo y prueba en laboratorio de un circuito rectificador de alimentación monofásica, concebido para la alimentación de potencia eléctrica de sistemas UPS de hasta 5 $\mathrm{kW}$, y con la característica particular de contar con un esquema de reducción de contenido armónico en la señal de corriente de entrada, de simple implementación y alta velocidad de respuesta. El prototipo implementado se dotó con un pequeño y muy económico microcontrolador PIC12F675 de microchip como unidad central de control, y se diseñó el circuito de potencia para trabajar en el límite de operación de modo continuo, con la idea de mejorar la eficiencia del sistema. De igual manera, se implementó un esquema de control por histéresis como estrategia general para la reducción del contenido armónico, comparando la corriente de entrada con una señal de referencia sinusoidal construida y sincronizada por el microcontrolador para la potencia y el voltaje nominal de salida. Con el fin de verificar la funcionalidad y el desempeño del esquema de diseño, se realizaron simulaciones del circuito de potencia y de su control, y se construyó un prototipo de laboratorio de $300 \mathrm{~W}$. En ambos casos, se observó un excelente comportamiento tanto en respuesta dinámica como en reducción de contenido armónico. 


\section{con-ciencias}

\section{Abstract}

This paper presents the design, development and laboratory testing of a single-phase rectifier, designed for electric power supply of UPS systems up to 5 $\mathrm{kW}$, with the particular characteristic to count on a scheme of reduction of harmonic content in the input current, of simple implementation and high speed response. The implemented prototype was equipped with a small and inexpensive Microchip PIC12F675 microcontroller as the central control unit, and the power circuit designed to work in the limit of continuous mode operation, with the idea of improving the efficiency of the system. A scheme of hysteresis control like general strategy for the reduction of the harmonic content was implemented, comparing the input current to a sinusoidal reference signal constructed and synchronized by the microcontroller to the power and the rated output voltage. In order to verify the functionality and performance of the design scheme, simulations of the circuit of power and their control were realized, and a laboratory prototype of $300 \mathrm{~W}$ was constructed. In both cases, an excellent behavior in dynamic answer and reduction of harmonic content was observed.

\section{Introducción}

En la actualidad, es posible observar que el desarrollo tecnológico de la industria electrónica de potencia está encaminado a la obtención de la máxima eficiencia y rendimiento, lo cual se refleja en la búsqueda de una mayor velocidad de conmutación y de un menor tamaño de empaque desde el punto de vista de los dispositivos y en un aumento de la eficiencia energética desde el punto de vista del sistema. Otro fenómeno observado es que la mayor parte de las cargas conectadas a la red son de origen electrónico, éstas utilizan, en media y baja potencia, una fuente de alimentación monofásica convencional. Esta clase de aparatos trabajan normalmente con un bajo Factor de Potencia (FP) y una alta distorsión armónica, utilizando inadecuadamente la energía eléctrica (flujo de corrientes armónicas que no aportan potencia útil).

Debido al FP tan bajo de estos equipos, las pérdidas de energía son muy altas y la distorsión armónica generada es transmitida a la línea de alimentación, lo cual causa fallas de funcionamiento en equipos muy sensibles, entre otros problemas documentados en la bibliografía técnica $[1,2]$.
Teniendo en cuenta las condiciones presentadas en la red de potencia eléctrica colombiana, este proyecto propone diversas ideas para solucionar este tipo de problemas. En particular, se considera la condición presentada por ciertos equipos para el acondicionamiento de la energía eléctrica UPS (Uninterruptible Power Supply, Sistema de Alimentación Ininterrumpida) que se han masificado en el mercado nacional en los últimos años [3, 4, 5]. Muchos de estos equipos se producen en el país, compitiendo con los importados a través de distribuidores especializados. Este mercado de las UPS se puede caracterizar en rangos de potencia: los de alimentación monofásica y bifásica (potencias de $8 \mathrm{kVA}$ máximo) y los de alimentación trifásica (potencias superiores a los 8 kVA). En ambos casos, se requiere de un circuito rectificador de alimentación, cuyo principal objetivo, luego de entregar el voltaje requerido regulado, es proporcionar condiciones óptimas para el uso de la red de alimentación desde el punto de vista de factor de potencia y distorsión armónica.

En la mayoría de los países industrializados se procesa la energía eléctrica a través de convertidores electrónicos de potencia, los cuales tienen como función adaptar las condiciones del suministro, elevar el aprovechamiento de la energía y, a su vez, optimizar el rendimiento del equipo. La estructura 


\section{con-ciencias}

variable que caracteriza a los convertidores electrónicos de potencia ha exigido fuertes desarrollos en los esquemas de control, explorando con mucho éxito el uso de dispositivos microcontrolados $[6,7$, $8,9,10]$. Este tipo de tendencias también se han detectado en las nuevas propuestas de control en convertidores monofásicos de media potencia.

Este trabajo se proyectó para desarrollar un sistema de rectificación monofásica para alimentación de sistemas (UPS) con corrección activa de factor de potencia, con una potencia nominal de $5[\mathrm{~kW}]$; el diseño fue evaluado satisfactoriamente por simulación y sobre un prototipo de laboratorio de 300 [W]. Además, se desarrolló un circuito de control microcontrolado autoprotegido que garantiza una salida estable y conforme con los requerimientos de los equipos a los que les suministra alimentación.

\section{Rectificación monofásica y corriente de alimentación}

El circuito de potencia de entrada para equipos electrónicos, como es el caso de las UPS, está compuesto por lo general de un condensador de entrada, seguido de un circuito rectificador en puente completo. Dado este tipo de circuito con comportamiento no lineal, la entrada de corriente resultante termina presentando muchos componentes armónicos de bajo orden (Figuras 1 y 2).
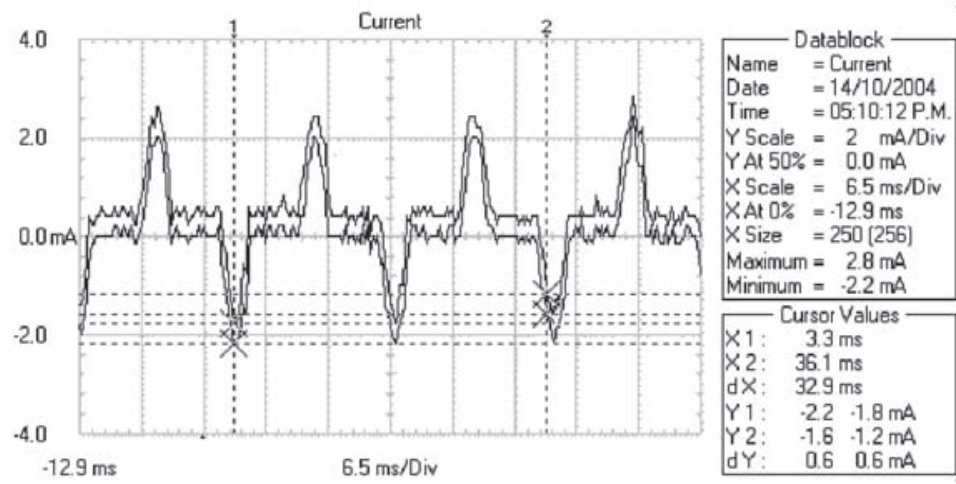

Figura 1. Corriente de entrada de un rectificador monofásico sin control de contenido armónico en su entrada.

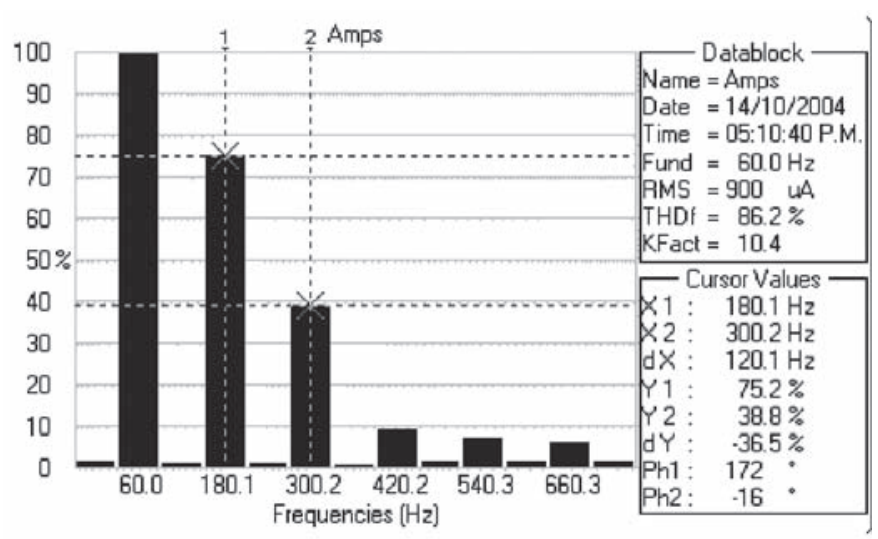

Figura 2. Contenido armónico de la corriente de entrada de un rectificador monofásico sin control de contenido armónico. 


\section{con-ciencias}

En la operación normal de una UPS, el voltaje de entrada AC (monofásico o trifásico, dependiendo de la potencia manejada por el circuito) es convertido en un voltaje regulado estable a través de un rectificador conmutable de alta frecuencia, y su potencia DC es alimentada al inversor PWM con el fin de generar la señal alterna ideal para las cargas sensibles (Figura 3).

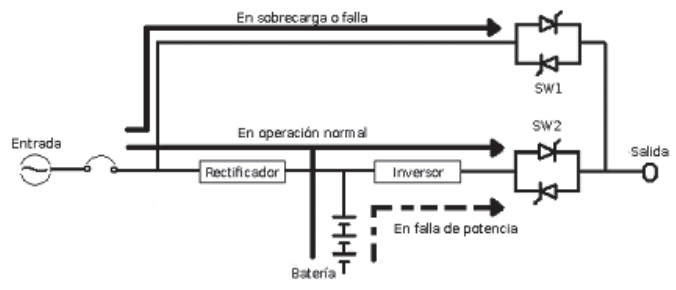

Figura 3. Diagrama esquemático de una UPS.

Lo ideal es que la entrada de corriente de la UPS sea controlada para mantener una forma de onda sinusoidal por medio de un rectificador conmutable o activo, con el fin de obtener una corriente de alta calidad, libre de exceso de corrientes armónicas.

\section{Metodología}

Para lograr la operación deseada, conservando una alta eficiencia en el circuito de potencia, se definieron dos criterios claves en el diseño del circuito rectificador elevador:

- Modo de operación y topología del circuito de potencia. Su definición incide directamente en el diseño y selección de los componentes del circuito de potencia. A fin de lograr un alto desempeño, se optó por trabajar el circuito en modo de conducción crítica o por lo menos muy cerca de esta zona, como estrategia para el aumento de la eficiencia del circuito y para el manejo de la corriente de entrada para reducir el contenido armónico. En estas condiciones de operación, la corriente en el choque cae rápidamente a cero, lo que reduce su valor promedio (eficiencia) y permite manejar una envolvente con la forma de onda deseada (corrección de factor de potencia). Es claro que esta estrategia de diseño genera sobre la corriente un ruido de alta frecuencia, pero por su característica puede ser reducido con un pequeño filtro capacitivo en la entrada. Adicionalmente, se trasladó el choque del circuito $L_{1}$ al lado $\mathrm{AC}$ antes del rectificador, con el objetivo de ayudar en la reducción del ruido de la conmutación.

- Esquema de control del circuito. Dado que el principal objetivo del circuito de rectificación es mantener una onda de corriente con forma cercana a la sinusoidal y en fase con el voltaje de línea, se implementó un control por histéresis en donde se comparara la corriente de entrada con una banda seno construida en la unidad de control a partir de los valores del voltaje de salida y de potencia de salida del convertidor (Figura 4).

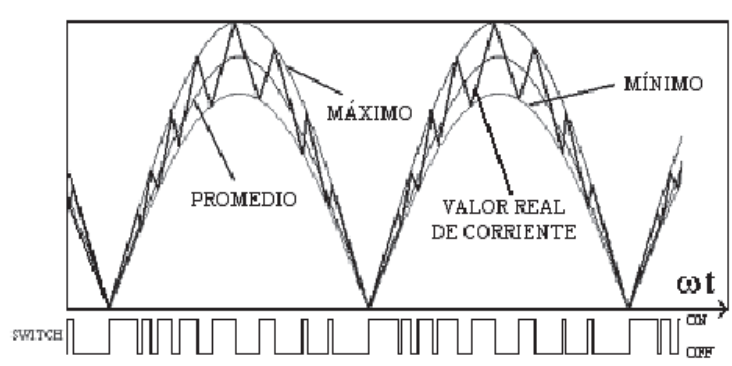

Figura 4. Detalle del esquema de control por histéresis.

Esta estrategia de control se basa en la continua comparación de la corriente de entrada (voltaje sobre la resistencia sensora $R_{S}$ ) con un valor de corriente de referencia construido a partir de la potencia de salida y la forma de onda deseada (onda seno en fase con el voltaje de línea). La forma de onda fue memorizada dentro de la memoria ROM del microcontrolador.

Esta opción de control requiere conocer con exactitud los instantes de cruce por cero para sincronizar 


\section{con-ciencias}

la señal. Así, el microcontrolador implementa un simple circuito detector de cruce por cero basado en sus comparadores internos. La señal de voltaje de línea (realmente la secundaria de un pequeño transformador aislado) se reduce a niveles seguros para el microcontrolador a través de un divisor resistivo, cuyo valor se determina asegurando sobre el microcontrolador un voltaje máximo de 2,5 $\mathrm{V}$ y un flujo de corriente del orden de los miliamperios (Figura 5).

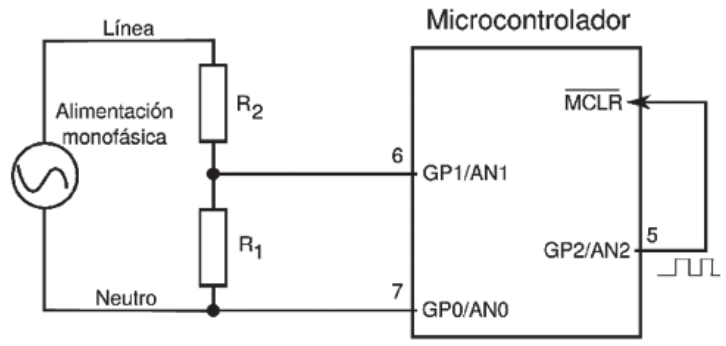

Figura 5. Circuito detector de cruce por cero.

Los voltajes de neutro y proporcional de línea se alimentan para su comparación en los pines 7 (GP0/AN0) y 6 (GP1/AN1) del microcontrolador respectivamente, obteniéndose en el pin 5 (GP2/ AN2) una señal de $5 \mathrm{~V}$ cada vez que el voltaje en el pin $6\left(V_{a}\right)$ sea inferior al voltaje en el pin $7\left(V_{N}\right)$, teniéndose así una señal digital en alto en cada semiciclo de red. Esta señal es alimentada nuevamente al microcontrolador a la interrupción externa (MCLR) con el fin de sincronizar la operación del comparador de corriente.

De forma similar, se da la posibilidad a la unidad de control de leer el voltaje de salida escalando su valor con otro divisor resistivo $\left(R_{1}\right.$ y $R_{2}$ en la Figura $6)$, cuyo valor se determina de igual manera.

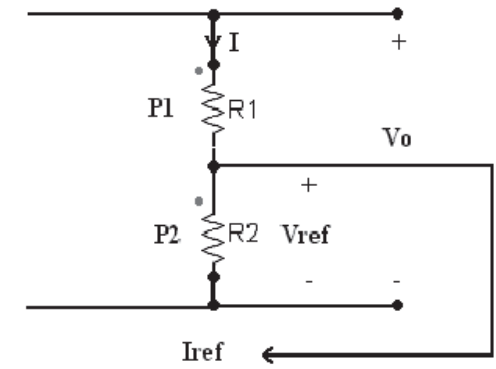

Figura 6. Sensor del voltaje de salida DC.

Para la lectura de la corriente de entrada $I_{L}$, se instala en la parte inferior del circuito una resistencia sensora $R_{S}$ de bajo valor, a través de la cual circula la corriente total del circuito y se produce sobre ella una caída de tensión proporcional a la corriente, que se puede acondicionar y entregar para su lectura al microcontrolador (Figura 7).

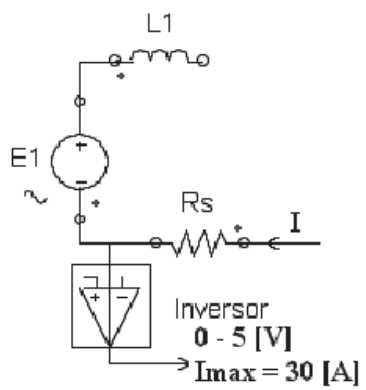

Figura 7. Lectura de la corriente de entrada.

El voltaje sobre esta resistencia sensora es invertido en polaridad utilizando un amplificador operacional (LM324), y luego leído por el convertidor A/D del microcontrolador, digitalizado a ocho bits para facilitar la comparación con los datos en memoria.

La idea general del control se basa en la comparación de la corriente de entrada (sensada a través de la resistencia sensora) con una corriente de referencia construida por el microcontrolador. Si la referencia se encuentra por encima de la corriente de entrada, el microcontrolador automáticamente dispara el tran- 


\section{con-ciencias}

sistor de potencia para aumentar el nivel de corriente. $\mathrm{Si}$, por el contrario, la referencia se encuentra por debajo de la corriente de entrada, el microcontrolador apaga el transistor con el fin de que la energía del convertidor se reduzca, al igual que la corriente de entrada. Este esquema permite control inmediato de la potencia de entrada y facilita la incorporación por software de una protección de sobrecorriente.

De acuerdo con estos criterios de diseño, se generaron las siguientes especificaciones nominales del circuito propuesto para una aplicación de $5 \mathrm{~kW}$ :

- El rectificador tendrá una salida de tensión nominal de $400 \mathrm{~V}$ DC.

- La tensión nominal de la fuente AC de entrada será de $120 \mathrm{~V}$ RMS, con un rango operativo de $\pm 30 \%$.

- La carga nominal resistiva será de $32 \Omega$.

- La frecuencia de conmutación será de 25 $\mathrm{kHz}$. Este valor es ideal teniendo en cuenta la frecuencia de operación del control dentro del microcontrolador. Sólo se tomará en cuenta para hacer los cálculos de los componentes del sistema, ya que el control de la planta se hará por histéresis y no existirá una frecuencia de conmutación como tal.

La Figura 8 muestra el diseño del circuito rectificador para una potencia nominal de $5 \mathrm{~kW}$.

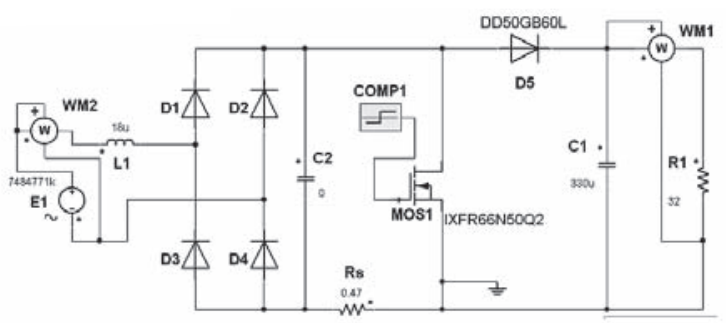

Figura 8. Circuito de potencia. Diseño a $5 \mathrm{~kW}$.

\section{Resultados}

Se realizó la simulación del circuito de potencia (Figura 8) y su esquema de control, considerando las limitaciones relacionadas con la velocidad del microcontrolador. En la Figura 9 se observa el comportamiento de la corriente de entrada cuando el convertidor opera a plena carga.

A primera vista, los resultados por simulación parecen validar el circuito de potencia, su diseño y la estrategia de control. El circuito es capaz de alimentar la potencia nominal con una eficiencia medida promedio del $82,2 \%$ y una distorsión armónica sobre la corriente de entrada del 16,3\%.

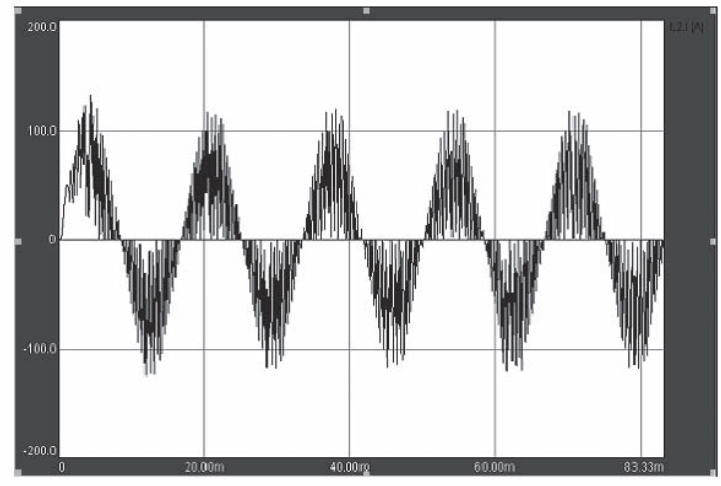

Figura 9. Corriente de entrada. Simulación rectificador de $5 \mathrm{~kW}$.

\section{Resultados prototipo de laboratorio}

Con el propósito de evaluar el desempeño del circuito propuesto y su control, se diseñó e implementó un prototipo de laboratorio equivalente al diseño simulado con una potencia nominal de 300 W. El perfil definido para este prototipo escalado en potencia es el siguiente:

- Potencia nominal de salida de $300 \mathrm{~W}$.

- El rectificador tendrá una salida de tensión nominal de $250 \mathrm{~V}$ DC. 
- La tensión nominal de la fuente AC de entrada será de $90 \mathrm{~V}$ RMS, con un rango operativo de $\pm 30 \%$.

- La carga nominal resistiva será de $208 \Omega$.

- La frecuencia de conmutación será de 25 $\mathrm{kHz}$. Este valor es ideal teniendo en cuenta la frecuencia de operación del control dentro del microcontrolador. Sólo se tomará en cuenta para hacer los cálculos de los componentes del sistema, ya que el control de la planta se hará por histéresis y no existirá una frecuencia de conmutación como tal.

- Los componentes del circuito de potencia se calcularon siguiendo los mismos criterios y expresiones algebraicas utilizadas para el diseño del circuito de $5 \mathrm{~kW}$, los mismos que respetan los criterios del convertidor, del esquema de control propuesto y las características de la unidad de control a utilizar.

En la Figura 10 se puede observar el diseño final y selección de componentes del circuito a $300 \mathrm{~W}$.

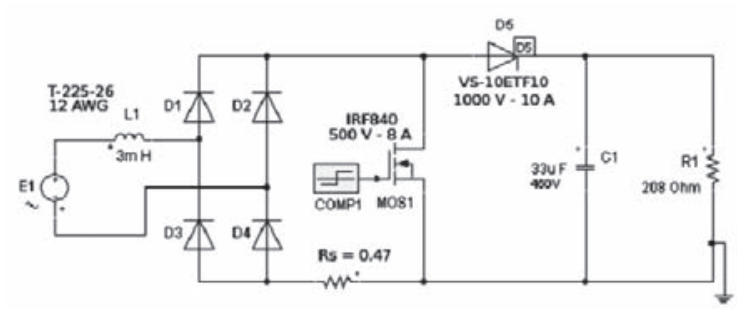

Figura 10. Circuito de potencia. Diseño a $300 \mathrm{~W}$.

La Figura 11 presenta en la parte inferior la corriente de entrada medida sobre el prototipo de laboratorio de $300 \mathrm{~W}$ operando a plena carga (la curva superior pertenece al voltaje de alimentación alterna, el desfase de $180^{\circ}$ se debe a la posición de la pinza de corriente). Como se observa, existe una gran similitud de forma de onda entre la corriente real y la corriente simulada. Sin embargo, la distorsión armónica me- dida en el circuito real sí fue sensiblemente mayor, de $21,2 \%$ (Figura 12), esto debido a las condiciones reales de operación del circuito.

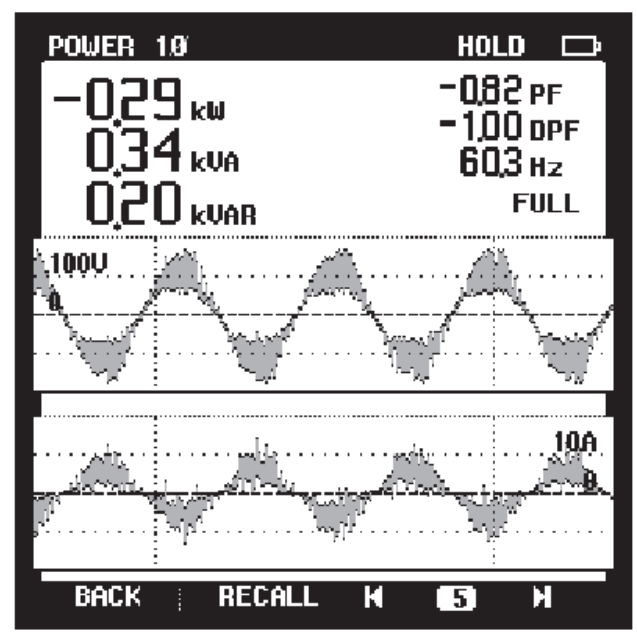

Figura 11. Voltaje y corriente de entrada con valores de factor de potencia, factor de desplazamiento y potencias. Prototipo de laboratorio de $300 \mathrm{~W}$.

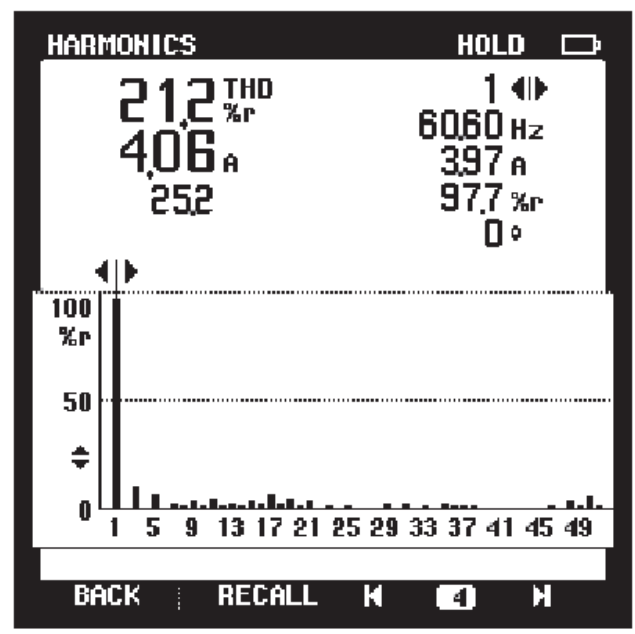

Figura 12. Contenido armónico de la corriente de entrada. Prototipo de laboratorio de $300 \mathrm{~W}$.

De igual forma, se evaluó el comportamiento dinámico del circuito frente a cambios en el voltaje de entrada (se varió la alimentación alterna entre 


\section{con-ciencias}

70 y 120 voltios eficaces) y en la carga resistiva de salida (valores de carga entre $150 \Omega$ y $270 \Omega$ ), observándose en todos los casos una rápida regulación del voltaje de salida DC.

\section{Conclusiones}

Se logró demostrar la hipótesis inicial respecto al diseño del convertidor, concretamente del choque de entrada, operando en modo continuo pero muy cerca de la condición crítica con la idea de reducir el consumo de corriente y aumentar la eficiencia del circuito. Esta reducción fue causada por la disminución de la corriente promedio en el lazo de entrada, lo que ocasionó una reducción en las pérdidas en este lazo, con la ventaja adicional de permitir eliminar el sobrepico de corriente en el arranque con un control adecuado (rápido y correctamente sintonizado).

La segunda hipótesis, relacionada con la reducción de contenido armónico en la corriente de entrada al controlar el comportamiento promedio de la corriente discontinua de entrada, también se logró validar. En este caso, el control por comparación con una señal de referencia permitió una respuesta rápida y una implementación simple sobre un microcontrolador de muy bajo costo.

Con la idea de validar el diseño, se construyó un prototipo de $300 \mathrm{~W}$, siguiendo los principios de diseño derivados del análisis de operación del circuito, y se realizaron simulaciones tanto al circuito de $300 \mathrm{~W}$ como al circuito objetivo de $5 \mathrm{~kW}$. Los resultados obtenidos en ambos casos permitieron validar la hipótesis de diseño, observándose un circuito regulador de voltaje DC con muy bajo contenido armónico en la corriente de entrada, sencillo de implementar y de muy bajo costo.

\section{Agradecimientos}

Los autores desean dar las gracias al semillero de investigación SIEPOT por sus contribuciones en la construcción del prototipo experimental, al grupo de investigación ARMOS por su apoyo en el desarrollo, al CIDC de la Universidad Distrital por la financiación del proyecto institucional que enmarca el trabajo y a los revisores por sus valiosas sugerencias para mejorar el documento.

\section{Referencias bibliográficas}

[1] G. Nicholson, V. Gosbell, A. Parsotam, "Analysis of Harmonic Distortion Levels on a Distribution Network", Universities Power Engineering Conference, pp. 1-7. 2007, AUPEC 2007, Australasian.

[2] C. Demoulias, D. Goutzamanis, K. Gouramanis, "Voltage Harmonic Distortion at Buses Feeding Office Loads", Power Tech, 2007 IEEE Lausanne, pp. 1546-1551, julio 2007.

[3] W. Solter, "A New International UPS classification by IEC 62040-3",
Telecommunications Energy Conference, INTELEC. 24th Annual International, pp. 541-545, 2002.

[4] S. Skok, M. Skok, N. Vrkic, "Electrical Performance Test Procedure for Uninterruptible Power Supplies", Industrial Technology, IEEE ICIT '04. 2004, IEEE International Conference, vol.2, pp. 667-67, 2004.

[5] S. Chand, K. Chawla, "EMC Evaluation and Analysis of UPS", Electromagnetic Interference and Compatibility, Proceedings 
of the International Conference 21-23 Feb. 2002, pp. 37-42, 2002.

[6] Z. Jinghai, L. Zhengyu, R. Yuancheng, Q. Zhaoming, W. Yousheng, "Novel Sampling Algorithm for DSP Controlled $2 \mathrm{~kW}$ PFC Converter", Power Electronics, IEEE Transactions, pp. 217 - 222, marzo 2001.

[7] E. H. Miliani, D. Depernet, J. M. Kauffmann, "New Control Strategy and its DSP Implementation for a Naturally Commutated Matrix Converter in a Variable Speed Constant Frequency Generating System", European Conference on Power Electronics and Applications, p.10, 2005.

[8] D. He, R. M. Nelms, “Average Current-Mode Control for a Boost Converter using an 8-bit

\section{con-ciencias}

Microcontroller", 2004 IEEE International Symposium on Industrial Electronics, vol. 2, pp. 1185-1190, mayo 2004.

[9] W. Qian, Z. Dong-Lai, “All Digital DC/ DC Converters on FPGA", Intelligent Computation Technology and Automation (ICICTA), 2008 International Conference, vol 2, pp. 11-15, octubre 2008.

[10] F. H. Martínez S., D. F., Gómez M, "Fuzzy Logic Controller for Boost Converter with Active Power Factor Correction", Power Electronics, ICPE '07. 7th Internatonal Conference on, pp. 936-940, octubre 2007. 